\title{
Relation between delayed feedback and delay-coupled systems and its application to chaotic lasers
}

\author{
Miguel C. Soriano, ${ }^{\text {a) }}$ Valentin Flunkert, and Ingo Fischer \\ Instituto de Física Interdisciplinar y Sistemas Complejos, IFISC (CSIC-UIB), Campus Universitat \\ Illes Balears, E-07122 Palma de Mallorca, Spain
}

(Received 22 March 2013; accepted 26 November 2013; published online 13 December 2013)

\begin{abstract}
We present a systematic approach to identify the similarities and differences between a chaotic system with delayed feedback and two mutually delay-coupled systems. We consider the general case in which the coupled systems are either unsynchronized or in a generally synchronized state, in contrast to the mostly studied case of identical synchronization. We construct a new time-series for each of the two coupling schemes, respectively, and present analytic evidence and numerical confirmation that these two constructed time-series are statistically equivalent. From the construction, it then follows that the distribution of time-series segments that are small compared to the overall delay in the system is independent of the value of the delay and of the coupling scheme. By focusing on numerical simulations of delay-coupled chaotic lasers, we present a practical example of our findings. (C) 2013 AIP Publishing LLC. [http://dx.doi.org/10.1063/1.4844335]
\end{abstract}

Dynamical systems with time delay exhibit a rich variety of different behaviors, including the possibility of highdimensional chaotic behavior. Although a lot of insight has been gained on the principles of delay-coupled systems, a direct relation between the properties of a system formed by a single nonlinear element subject to delayed self-feedback and a system of two identical mutually delayed-coupled elements is still lacking. Since these two systems share a number of features, it is intriguing to elaborate further on the mechanisms that give rise to this similar emerging behavior. We show to which degree the statistical properties of the chaotic attractor of a delaycoupled system for a given parameter set are preserved when comparing a system with a single element with selffeedback and a system with two mutually delay-coupled elements. By using simple construction rules, we illustrate how the statistical properties of the underlying attractor are preserved. For illustration purposes, we focus on numerical simulations of semiconductor lasers with optical feedback as a paradigmatic example of delay-coupled systems.

\section{INTRODUCTION}

Many nonlinear dynamical systems show chaotic behavior for a wide range of parameters. The chaotic motion of the system occurs around strange attractors, ${ }^{1-3}$ with a strong dependence on the initial conditions. This sensitivity to the initial conditions is characterized by one or more positive Lyapunov exponents. Low-dimensional systems have a small number of positive exponents, and the characterization of its attractor is relatively easy with embedding techniques. ${ }^{4}$ In contrast, high-dimensional systems may have a large number of positive Lyapunov exponents making the characterization of the attractor more complicated.

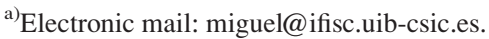

Systems ruled by delay differential equations are important examples for such high dimensional systems and have received a lot of interest since the seminal works of Mackey and Glass ${ }^{5,6}$ and Ikeda. ${ }^{7}$ For delay systems, the phase space is infinite dimensional, i.e., the state of the system at a time $t$ is described by a function over the interval $[t-\tau, t]$ with $\tau$ being the delay time. Delay systems have been widely studied due to their peculiar properties as well as their importance in engineering, biology, and physics. ${ }^{8}$

Systems with delayed feedback and/or coupling are interesting dynamical systems, because they exhibit complex behavior despite their apparent simplicity. One approach to better understand the complex behavior of delayed systems is to focus on statistical aspects of the dynamics. Recently, Van der Sande et al. ${ }^{9}$ numerically showed that the autocorrelation function of a single nonlinear element with delayed self-feedback is related to the autocorrelation function of a unidirectionally delay-coupled ring configuration with an arbitrary number of elements $(N)$ via a scaling relation in the case of chaotic dynamics: The peak at $t=N \tau$ in the autocorrelation function of a single element with self-feedback of delay time $\tau$ is identical to the peak at $t=\tau$ in the autocorrelation function of a unidirectionally coupled ring with $N$ elements with overall delay time $\tau$.

But how exactly are the chaotic attractors of a single system with delayed self-feedback and of multiple systems with delayed-coupling related?

In this work, we provide new insights to this question by analyzing statistical properties of the chaotic attractors of a single system with delayed self-feedback and two systems with delayed coupling. In particular, we explain the origin of the scaling relation found by Van der Sande et al. ${ }^{9}$ for chaotic dynamics of delay-coupled Ikeda oscillators and semiconductor lasers, and we conjecture that time-series segments that are short compared to the overall delay have the same probability to occur in either system. That is, the two setups are indistinguishable when they are observed on time-scales shorter than the delay time. 
We further conjecture that the evolution of such a timeseries segment through the setup has the same statistics in both cases, i.e., the response of a laser to the segment entering as the drive is statistically identical. This leads to equivalent long range correlations for the two setups in addition to the equal short range statistics.

We test this conjecture by comparing the two configurations shown in Fig. 1: a single system with self-feedback with delay $2 \tau$ (configuration I) and two systems mutually coupled with coupling delay $\tau$ (configuration II). For these two configurations, we, respectively, construct new time-series by concatenating time-series segments of length $\tau$ and show that, when the delay is large, these constructed time-series have identical statistical properties (not only identical correlations).

One particular consequence of this finding is that timeseries segments of length $\tau$ from configuration I and configuration II have identical probability distributions. That is, by only looking at segments of length $\tau$, individually, one cannot decide from which of the two systems they stem. Our results are a general feature of delay systems and can be directly extended to rings of unidirectionally delay-coupled elements with an arbitrary number of elements.

For concrete numerical simulations, we focus on semiconductor lasers with optical self-feedback as a paradigmatic model for systems with delayed self-feedback and delayinduced chaos. ${ }^{10}$ Over the last two decades, the study of optical and opto-electronical systems with delay has been a central topic of research in nonlinear science. There are two main reasons for this popularity: First, theoretical findings can be put to a test in a controllable practical environment using these systems. ${ }^{11-13,15-17}$ Second, delayed optical and opto-electronic systems are viable for many practical applications. ${ }^{18-21}$

\section{NUMERICAL AND ANALYTICAL RESULTS}

In the following, we compare the statistical properties of a semiconductor laser with self-feedback and two delaycoupled semiconductor lasers, as illustrated in Fig. 1. The lasers are single mode lasers with moderate delayed feedback (coupling) and operate in the chaotic regime. We investigate these two systems using an extension of the widely used Lang-Kobayashi rate equations. ${ }^{13}$ The rate equations for the complex slowly varying amplitude of the electric field $E_{i}(t)$ and the carrier number inside the cavity $N_{i}(t)$ for laser $i$ in a delay-coupled configuration are given by

$$
\begin{gathered}
\dot{E}_{i}(t)=\frac{1+i \alpha}{2}\left[G_{i}(t)-\frac{1}{\tau_{p}}\right] E_{i}(t)+\gamma E_{j}\left(t-\tau_{c, f}\right) e^{-i \Phi}, \\
\dot{N}_{i}(t)=\frac{J}{e}-\frac{N_{i}(t)}{\tau_{N}}-G_{i}(t)\left|E(t)_{i}\right|^{2},
\end{gathered}
$$

I)

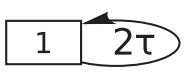

II)

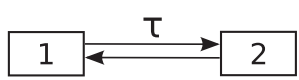

FIG. 1. Scheme of a system with delayed self-feedback (configuration I) and two systems in a mutually delay-coupled configuration (configuration II). where $G_{i}(t)=g\left(N_{i}(t)-N_{0}\right) /\left(1+s I_{i}(t)\right)$ is the optical gain and $I_{i}(t)=\left|E_{i}(t)\right|^{2}$ is the intensity of laser $i$. For a single laser with feedback $i=j=1$ and for two mutually coupled lasers $(i, j)=(1,2)$ and $(i, j)=(2,1)$, respectively. The feedback (coupling) delay time was chosen as $\tau_{f}=4 \mathrm{~ns}$ ( $\tau_{c}=2 \mathrm{~ns}$ ). Parameter $\alpha=5$ is the linewidth enhancement factor, $\tau_{p}=2 \mathrm{ps}$ is the photon lifetime, $\tau_{N}=2 \mathrm{~ns}$ is the carrier lifetime, $g=1.5 \times 10^{-8} \mathrm{ps}^{-1}$ is the differential gain coefficient, $N_{0}=1.5 \times 10^{8}$ is the carrier number at transparency, $s=5 \times 10^{-7}$ is the gain compression coefficient, $\gamma=20 \mathrm{~ns}^{-1}$ is the feedback/coupling strength, $\Phi=0$ is the optical feedback phase, and $e$ is the electron charge. The parameter $J$ denotes the bias current and was chosen as $J=2 J_{t h}$, where $J_{t h}=14.7 \mathrm{~mA}$ is the threshold current. For this set of parameters, the solitary laser relaxation oscillation (RO) period is $T_{R O}=0.17 \mathrm{~ns}$. In the following, we consider the long delay limit ${ }^{14}$ and choose a delay time of $2 \mathrm{~ns}$, which is an order of magnitude larger than $T_{R O}$.

We present numerical simulations, along with analytical arguments to sustain our conjecture. In a more general framework, we consider a single system with delayed selffeedback (configuration I) described by

$$
\dot{x}(t)=f[x(t), x(t-2 \tau)] .
$$

The single system with self-feedback with delay $2 \tau$ defined in Eq. (3) can be used as the generator of two different sequences of time-series that each corresponds to a single system with self-feedback with delay $\tau$. The definition of two complementary time-series constructed from configuration I enables a more evident comparison between configurations I and II. Therefore, we define two new variables by splitting each time interval of length $2 \tau$ into two segments of length $\tau$. We then follow the evolution of each $\tau$-piece separately in steps of $2 \tau$. From these individual evolutions, we define two new time-series $y_{1}$ and $y_{2}$, where $y_{1}(t)$ is constructed by concatenating intervals $[0, \tau[,[2 \tau, 3 \tau[, \ldots$, and $y_{2}(t)$ is constructed by concatenating intervals $[\tau, 2 \tau[$, $[3 \tau, 4 \tau[, \ldots$ of the original variable $x(t)$

$$
\begin{aligned}
& y_{1}(t)=x(t+2 \tau\lfloor t / \tau\rfloor), \\
& y_{2}(t)=x(t+\tau+2 \tau\lfloor t / \tau\rfloor) .
\end{aligned}
$$

Here, $\lfloor\bullet\rfloor$ denotes the floor function (the largest integer not greater than the argument).

In these new variables, the dynamical equations are given by

$$
\dot{y}_{i}(t)=f\left[y_{i}(t), y_{i}(t-\tau)\right],
$$

however, due to the continuity of the original dynamics $x(t)$, the dynamics of $y_{1}$ and $y_{2}$ are discontinuous: each of the systems $y_{i}$ starts a new $\tau$-interval with a new initial value $y_{i}(n \tau)$ dictated by the other variable

$$
\begin{aligned}
& y_{1}(n \tau)=y_{2}(n \tau-0), \\
& y_{2}(n \tau)=y_{1}((n+1) \tau-0) \quad(n=0,1, \ldots) .
\end{aligned}
$$

It is important to distinguish between the history function and the initial value of a delay differential equation: The 
history function of $y_{i}$ is determined from the previous $\tau$ interval of $y_{i}$; however, the initial value $y_{i}(n \tau)$ is modified at the beginning of each delay interval.

Let us now address the delay coupled system (configuration II)

$$
\begin{aligned}
& \dot{x}_{1}(t)=f\left[x_{1}(t), x_{2}(t-\tau)\right], \\
& \dot{x}_{2}(t)=f\left[x_{2}(t), x_{1}(t-\tau)\right] .
\end{aligned}
$$

Similar to the analysis above, we introduce new coordinates by concatenating time-series of system 1 and 2 to form two new variables

$$
\begin{aligned}
& y_{1}(t)=\chi_{A}(t) x_{1}(t)+\chi_{B}(t) x_{2}(t) \\
& y_{2}(t)=\chi_{B}(t) x_{1}(t)+\chi_{A}(t) x_{2}(t),
\end{aligned}
$$

where

$$
\begin{aligned}
& A=\bigcup_{n \text { even }}[n \tau,(n+1) \tau[, \\
& B=\bigcup_{n \text { odd }}[n \tau,(n+1) \tau[,
\end{aligned}
$$

and $\chi_{A}$ and $\chi_{B}$ are the characteristic or indicator functions of these sets, i.e., $\chi_{A}(t)$ is unity if $t \in A$ and zero otherwise. Note that $A \cup B=\mathbb{R}$ and $A \cap B=\varnothing$. Similar to the case of configuration I, $y_{1}(t)$ now corresponds to following a timeseries segment of length $\tau$ as it travels between system 1 and system 2 as depicted in Fig. 2. From the illustration shown in Fig. 2, it follows that a complementary time-series, $y_{2}(t)$, can be constructed by following a time-series segment of length $\tau$, initially generated by system 2 , at $[0, \tau]$. Therefore, the two mutually delay-coupled systems can be used to construct two complementary chaotic trajectories of a system with self-feedback with delay $\tau$. The instantaneous trajectories of the two constructed variables $y_{1}(t)$ and $y_{2}(t)$ are not identical, but they are statistically equivalent.

For the variables constructed from the coupled system, the dynamical equations are then given also by Eq. (4)

$$
\dot{y}_{i}(t)=f\left[y_{i}(t), y_{i}(t-\tau)\right]
$$

And, again, due to the continuity of $x_{1}$ and $x_{2}$, the variables $y_{i}$ start with new initial values $y_{i}(n \tau)$ at each new delay interval

$$
\begin{aligned}
& y_{1}(n \tau)=y_{2}(n \tau-0), \\
& y_{2}(n \tau)=y_{1}(n \tau-0) .
\end{aligned}
$$

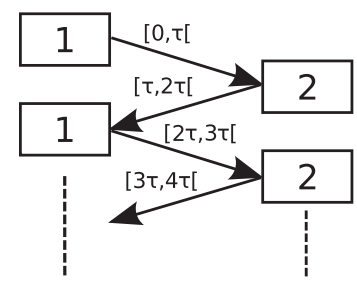

FIG. 2. Construction of a new time-series by following the evolution of a time-series segment of length $\tau$ through the two mutually delay-coupled systems (conf. II).
The only difference between configuration I and configuration II in this representation is the order in which the initial values are set between the two variables $y_{i}$ at each new delay interval (compare Eqs. (5) and (7)). We now show, numerically, that this difference in boundary conditions does not play a role for the statistical properties. That is, for a given history on a $\tau$-interval, the probability of finding a certain initial value in the next iteration is equal for time-series constructed from configurations I and II.

The independence of boundary condition is very similar to how spatially extended chaotic systems, such as the Belousov-Zhabotinsky reaction, behave. For these systems, it is known that the boundary conditions, e.g., square shaped or circular domain, become negligible for correlations and other statistical properties when the domain is large enough.

With this analogy in mind, the numerical results and arguments presented below can be expected. For our case, however, the representation through different boundary conditions is not obvious but only becomes apparent after the transformation.

Thus, this work presents new results on how a single node with delayed self-feedback is related to two nodes with mutual delayed coupling. ${ }^{10}$ We note that our considerations can be extended to more general network topologies, e.g., rings of unidirectionally coupled oscillators with delay. Whether it can be extended to further network configurations is an interesting question which would provide further insights into the behavior of a chaotic network in terms of a single unit.

A stricter mathematical proof of the equivalence between configurations I and II in the discussed sense lies beyond the scope of this manuscript. Such a proof is probably very challenging in the general case, since it would need to address the behavior of the natural measure of the two chaotic systems in the large delay limit and show a convergence to the same limit. It might however be feasible to show statistical equivalence for more simplified models, e.g., for simple chaotic maps like the Bernoulli map instead of a delay differential system, as has been done for the case of spatially extended systems. $^{22}$

The influence of the boundary conditions is illustrated in Fig. 3, where we plot the joint probability distributions of the laser intensities $p(I(t), I(t+2 \tau))$ for configuration I and $p\left(I_{1}(t), I_{2}(t+\tau)\right)$ for configuration II. We obtained Fig. 3 by simulating the respective system in a long time interval $\left(\left[0,10^{4} \tau\right]\right)$ (after transients have died out). From this long time-series, we sampled (with a time step of $d t=2 n s$ ) two data sets corresponding to $I(t)$ and $I(t+2 \tau)$ for the left plot and $I_{1}(t)$ and $I_{2}(t+\tau)$ for the right one, respectively. From these two data sets, we calculated the bi-dimensional normalized histogram using 100 bins for each axis. As shown in Fig. 3, the characteristic distributions for configurations I and II are very similar, even on the depicted logarithmic scale, implying the boundary conditions in Eqs. (5) and (7) are statistically equivalent.

This means, despite these differences in the precise definitions of the boundary conditions, the statistical properties of the constructed time-series are exactly the same when the delay time is large. Thus, in particular, time-series segments 

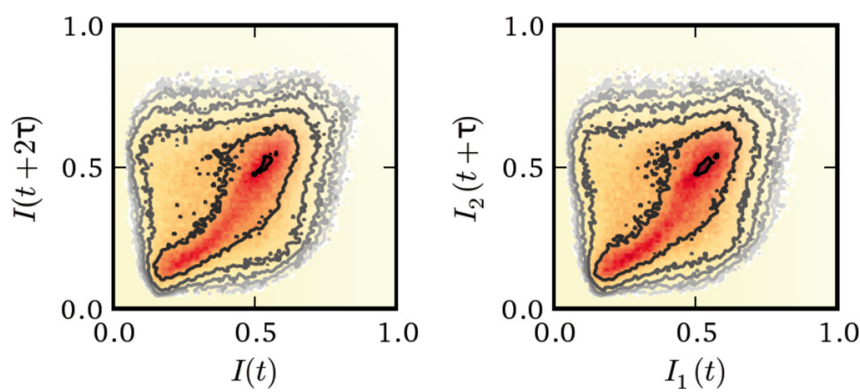

FIG. 3. Joint probability distributions of the intensities $p(I(t), I(t+2 \tau))$ for configuration I (left) and $p\left(I_{1}(t), I_{2}(t+\tau)\right)$ for configuration II (right). Darker colors indicate a higher density of occurrences in a logarithmic scale.

of length $\tau$ have the same probability to occur in the original configurations of two coupled systems with coupling delay $\tau$ and in the single system with delay time $2 \tau$. However, segments of constructed time-series longer than $\tau$ present single point discontinuities every $\tau$. While we find that these discontinuities do not alter the overall statistical equivalence between the two configurations, the complete equivalence between a laser with feedback and a time-series constructed from two delay-coupled lasers is no longer rigorously valid if segments longer than $\tau$ are considered.

Figure 4 shows the spatio-temporal plots ${ }^{23}$ of the two constructed time-series (the discontinuities are located on the $y$-axis). In this spatio-temporal representation, the delay time plays the role of a space variable, and subsequent $\tau$-segments are plotted on top of each other. ${ }^{23}$ The spatio-temporal plots in Fig. 4 show patterns that extend from one delay to the next with a temporal drift. These characteristic spatiotemporal patterns are identical in the two cases. This further illustrates the equivalence of the reconstructed time-series. By construction, the spatio-temporal patterns from the reconstructed time-series both match the spatio-temporal patterns of a single-laser with self-feedback with delay $\tau$.

As an additional illustration, we show in Fig. 5 the autocorrelation functions of the two constructed time-series for various feedback/coupling strengths. The autocorrelation functions show a narrow peak at $\mathrm{t}=0$ and additional

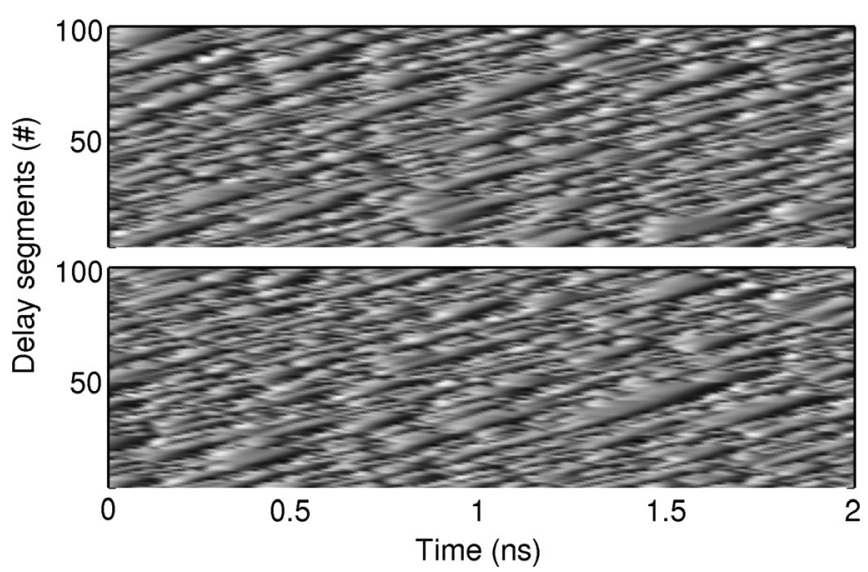

FIG. 4. Spatio-temporal representation of the constructed time series (e.g., $y_{1}$ ) from (top) the single laser with self-feedback (configuration I) and (bottom) the two coupled lasers (configuration II). $\tau=2 \mathrm{~ns}$. The intensity of the lasers is coded in a linear gray-scale, with darker colors indicating larger intensities. correlation peaks at multiples of the delay time. As it becomes evident in the insets of Figs. 5(a)-5(c), the autocorrelation functions completely match in all cases. In fact, we could not identify parameters within the chaotic regime for which the correspondence in terms of the autocorrelation function between time-series constructed from configurations I and II does not hold.

The autocorrelation functions shown in Fig. 5 correspond to time-series constructed from configurations I and II, respectively. In contrast, the properties of one of the original systems of configuration II are as follows, peaks occurring at even multiples of $\tau$ in Fig. 5 are the only ones present in the corresponding autocorrelation function. The peaks at odd multiples of $\tau$ are not present since there is no loop of length $\tau$ in this configuration. In fact, the peaks at odd multiples of $\tau$ appear in the cross correlations of the two elements in configuration II. $^{24}$

For delays sufficiently larger than the RO period, the constructed time-series from configurations I and II illustrate
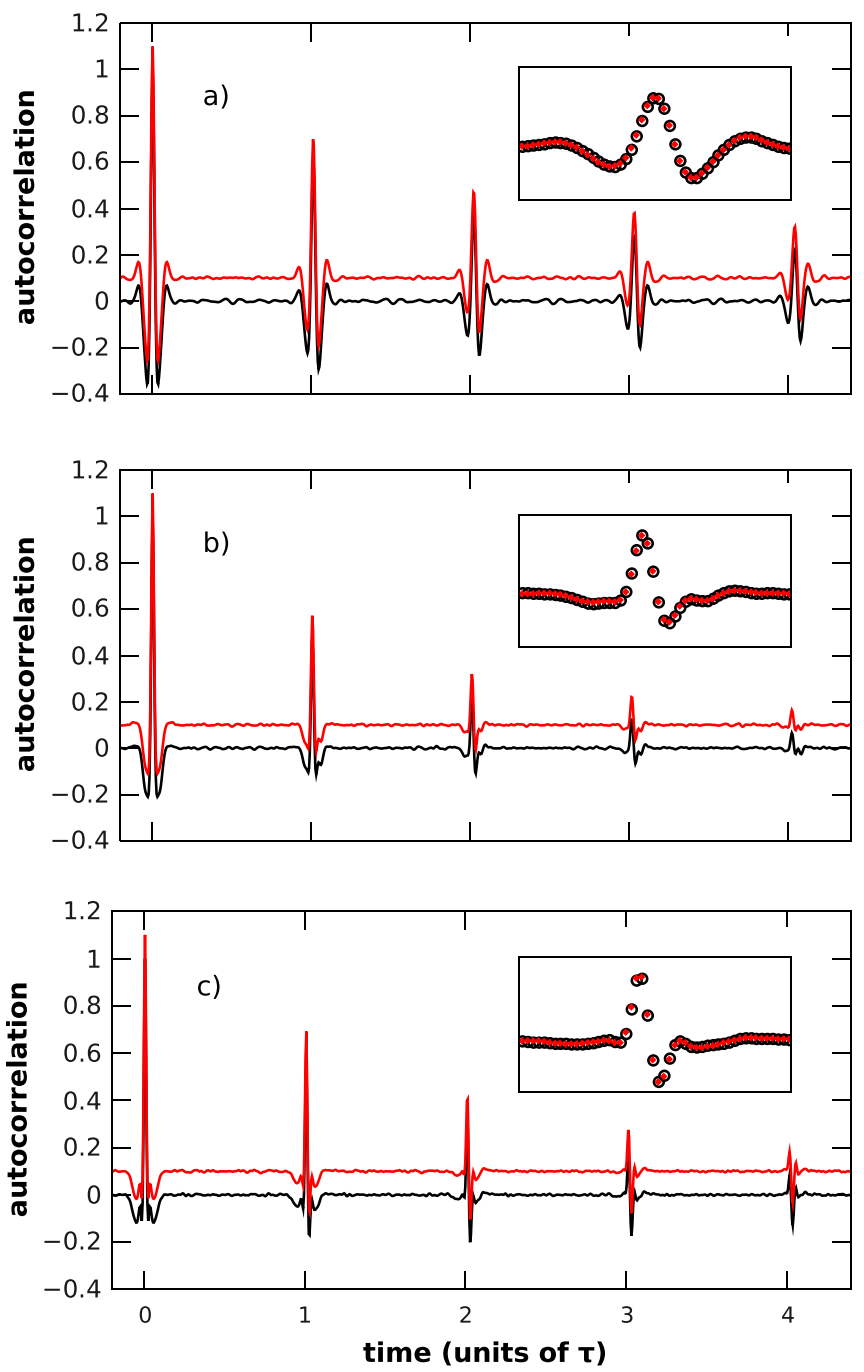

FIG. 5. Autocorrelation function of the constructed time-series of configuration I (black) and configuration II (red) for (a) $\gamma=20 \mathrm{~ns}^{-1}$, (b) $\gamma=40 \mathrm{~ns}^{-1}$, and (c) $\gamma=60 \mathrm{~ns}^{-1}$. The autocorrelation for configuration II is shifted up for easier comparison. Insets show a zoom of the (unshifted) autocorrelation peaks at time shift $2 \tau$, with (black) circles for configuration I and (red) diamonds for configuration II. 
that the shape and position of the autocorrelation peaks only depend on the number of times that each $\tau$-interval passes through a nonlinearity and the time between consecutive $\tau$-intervals. This explains the scaling relation found by Van der Sande et al. ${ }^{9}$ for rings of unidirectionally coupled oscillators with delay.

We provide significant evidence of the similarities between constructed time-series originating from a delayed feedback system and from a delay-coupled system. This also has implications for the long-standing question on how the time-series of the two delay-coupled lasers configurations compare to each other with respect to dimensionality and entropy. The time-series of laser 1 and laser 2 in configuration II relates to the evolution of consecutive delay-segments in a laser with feedback. These similarities between time-series from a delayed feedback system and from a delay-coupled system suggest that the configurations I and II have a similar dimensionality given they have the same overall delay. The entropy, since it is a rate, is expected to be double in configuration II as there are two statistically equivalent entropy sources for the same overall delay. As shown in Fig. 6(a), we have numerically checked that, in the long delay limit, the Kaplan-Yorke dimension of the single laser with delay feedback $2 \tau$ is similar to that one of the two coupled lasers system with coupling delay $\tau$. Furthermore, we have verified that the Kolmogorov-Sinai entropy of the laser with delay feedback $2 \tau$ is half the entropy of the two coupled lasers system with coupling delay $\tau$ (overall delay $2 \tau$ ) as illustrated in Fig. 6(b). The estimates of the Kaplan-Yorke dimension and the Kolmogorov-Sinai entropy require the prior computation of the spectrum of Lyapunov exponents. ${ }^{25}$ Complementary, in the case of two delay-coupled lasers with self-feedback, the particular dependence of the entropy and complexity measures on the coupling strength has been reported in Ref. 26 .

In the following, we analyze whether configurations I and II allow for the generation of identical trajectories in addition to having identical statistical properties. To that end, we need to prepare both configurations in a control state with the same initial history and initial conditions. By dividing the $2 \tau$-history of conf. I into two $\tau$-pieces, we can properly set the initial history in both configurations. Additionally, we can prepare laser 1 in conf. II with the same initial value of the laser in conf. I. However, we have

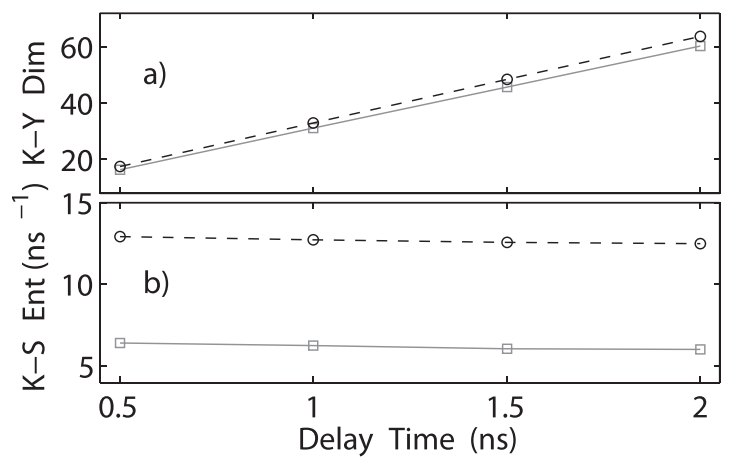

FIG. 6. (a) Kaplan-Yorke (K-Y) dimension and (b) Kolmogorov-Sinai (K-S) entropy for configurations I (grey solid line with squares) and II (black dashed line with circles) as a function of the overall delay time. no defined value for the initial condition of laser 2 in conf. II. This will inevitably cause a discontinuity in the timeseries generated from conf. II. As discussed below, the discontinuity in the time-series generated from conf. II will result in divergent trajectories after a certain time so that trajectories identical to conf. I cannot be generated.

In the chaotic regime, there are two qualitatively different behaviors in the trajectories constructed from configurations I and II when the systems are prepared in a control state. These two behaviors are related to the notion of weak and strong chaos. ${ }^{27,28}$ Figure 7 depicts the different behavior in these two regimes. A single laser subject to delayed feedback is simulated with the same history function, i.e., the same incoming drive, but two different initial values (red/ black). In the weak chaotic regime (panel a), the difference in initial conditions quickly decays and the response to the drive (the history) is the same after a short transient. However, the two trajectories start to diverge after a number of round-trips due to the perturbation produced by the different initial conditions (not shown). In contrast, if the dynamics is in the strong chaotic regime (panel b), the difference in initial conditions leads to two completely different responses instantly, despite the same drive.

This has the following implication: The perturbation produced by the discontinuity in conf. II grows in the length scale of $\tau$ for weak chaos, i.e., the trajectories will eventually diverge after a sufficiently large number of round-trips, while the perturbation grows within one $\tau$-segment for strong chaos and the trajectories start to diverge immediately. In summary, the trajectories constructed from configurations I and II diverge in two different time-scales depending on the particular chaos regime the lasers are operating in. While the strong and weak chaos regimes have a major influence on the synchronization properties of coupled lasers, ${ }^{27}$ our results hold for both chaos regimes. The joint intensity probability distribution, as illustrated in Fig. 3, remains constant for a given parameter set. This is also the case for the strong chaos regime. Therefore, the joint intensity probability distribution, which represents the response of the laser to an incoming drive (delayed self-feedback or coupling from another laser), is a relevant signature of the chaotic attractor.

We have shown that the statistical properties of the dynamics of a single laser with feedback, but not the exact trajectories, can be recreated by concatenating time-series

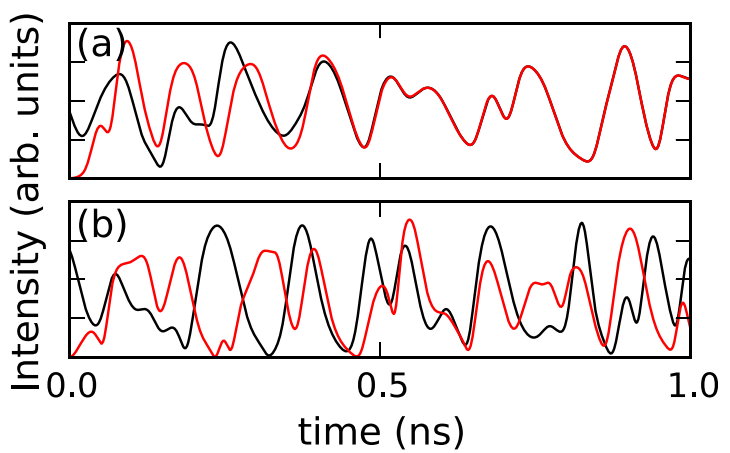

FIG. 7. Evolution of laser intensity over one delay interval with identical history but different initial conditions. (a) Consistent case (weak chaos); (b) inconsistent case (strong chaos). 
originating from a coupled system. Conversely, the same statistical properties of a laser in a mutually coupled configuration can be reconstructed from the dynamics of a laser with feedback with delay $2 \tau$. The constructed time-series would then be created by concatenating $2 \tau$-intervals of the laser with feedback in the range $t=[0,2 \tau[, t=[4 \tau, 6 \tau[, \ldots$ The autocorrelation of the time-traces created following this procedure would then only show the peaks at even multiples of the delay time in Fig. 5.

Finally, we note that we have numerically checked that our considerations also hold for various parameter sets of Ikeda and Mackey-Glass oscillators in the chaotic regime and long delays.

\section{CONCLUSION AND OUTLOOK}

We have presented strong numerical evidence indicating that chaotic time-series originating from systems with delayed self-feedback and delayed coupling have identical statistical properties over any given interval of length smaller than the delay. Thus, one cannot distinguish between the two time-series when only looking at segments of length smaller or equal than $\tau$.

Moreover, we provide a construction scheme generating two new time-series from the two configurations. By concatenating segments of length $\tau$, such that two consecutive segments in the constructed time-series have passed through the same number of nonlinear elements, new time-series are obtained. Using this construction, we show that the generated time-series are statistically indistinguishable.

Although the underlying chaotic attractors of the two configurations are different, the projections of the attractors that correspond to the constructed time-series are equal. These constructed time-series allow to infer the relationship between the dimensionality and entropy of the original systems. The entropy of the two delay-coupled lasers is twice the one of the single laser with delayed self-feedback, while the dimensionality of both systems is almost identical. How the attractor topology of the two configurations differs in detail remains an open question.

\section{ACKNOWLEDGMENTS}

We are grateful to Guy Van der Sande, Thomas Jüngling, Claudio R. Mirasso, Wolfgang Kinzel, Emilio Hernandez-Garcia, Daniel Brunner, and Pere Colet for fruitful discussions on the properties of delay-coupled systems. This work was supported by the MICINN (Spain), Comunitat Autònoma de les Illes Balears, FEDER, and European Commission under Projects TEC2012-36335 (TRIPHOP), TEC2009-14101 (DeCoDicA), FIS2007-60237 (FISICOS), $0200950 I 190$ (Proyecto Intramurales Especiales), Grups Competitius, and EC FP7 Project PHOCUS under Grant No. 240763. V.F. acknowledges financial support from the German Academic Exchange Service (DAAD).

${ }^{1}$ F. Takens, "Detecting strange attractors in turbulence," in Dynamical Systems and Turbulence, Lecture Notes in Mathematics Vol. 898, edited by D. A. Rand and L.-S. Young (Springer-Verlag, 1981), pp. 366-381.
${ }^{2} \mathrm{P}$. Grassberger and I. Procaccia, "Measuring the strangeness of strange attractors," Physica D 9, 189-208 (1983).

${ }^{3}$ J.-P. Eckmann and D. Ruelle, "Ergodic theory of chaos and strange attractors," Rev. Mod. Phys. 57, 617 (1985).

${ }^{4}$ A. Wolf, J. B. Swift, H. L. Swinney, and J. A. Vastano, "Determining Lyapunov exponents from a time series," Physica D 16, 285-317 (1985).

${ }^{5}$ M. C. Mackey and L. Glass, "Oscillation and chaos in physiological control systems," Science 197, 287-289 (1977).

${ }^{6}$ J. D. Farmer, "Chaotic attractors of an infinite-dimensional dynamical system," Physica D 4, 366-393 (1982).

${ }^{7} \mathrm{~K}$. Ikeda and K. Matsumoto, "High-dimensional chaotic behavior in systems with time-delayed feedback," Physica D 29, 223 (1987).

${ }^{8}$ T. Erneux, "Applied delay differential equations," in Surveys and Tutorials, Applied Mathematical Sciences Vol. 3 (Springer, 2009).

${ }^{9}$ G. Van der Sande, M. C. Soriano, I. Fischer, and C. R. Mirasso, "Dynamics, correlation scaling, and synchronization behavior in rings of delay-coupled oscillators," Phys. Rev. E 77, 055202(R) (2008).

${ }^{10}$ M. C. Soriano, J. García-Ojalvo, C. R. Mirasso, and I. Fischer, "Complex photonics: Dynamics and applications of delay-coupled semiconductors lasers," Rev. Mod. Phys. 85, 421-470 (2013).

${ }^{11}$ D. Lenstra, B. H. Verbeek, and A. J. den Boef, "Coherence collapse in single-mode semiconductor lasers due to optical feedback," IEEE J. Quantum Electron. 21, 674-679 (1985).

${ }^{12}$ I. Fischer, G. H. M. van Tartwijk, A. M. Levine, W. Elsäßer, E. Göbel, and D. Lenstra, "Fast pulsing and chaotic itinerancy with a drift in the coherence collapse of semiconductor lasers," Phys. Rev. Lett. 76, 220-223 (1996).

${ }^{13}$ T. Heil, I. Fischer, W. Elsäßer, J. Mulet, and C. R. Mirasso, "Chaos synchronization and spontaneous symmetry-breaking in symmetrically delaycoupled semiconductor lasers," Phys. Rev. Lett. 86, 795-798 (2001).

${ }^{14}$ T. Heil, I. Fischer, W. Elsäßer, and A. Gavrielides, "Dynamics of semiconductor lasers subject to delayed optical feedback: The short cavity regime,” Phys. Rev. Lett. 87, 243901 (2001).

${ }^{15} \mathrm{H}$. Fujino and J. Ohtsubo, "Synchronization of chaotic oscillations in mutually coupled semiconductor lasers," Opt. Rev. 8, 351-357 (2001).

${ }^{16}$ M. Nixon, M. Friedman, E. Ronen, A. A. Friesem, N. Davidson, and I. Kanter, "Synchronized cluster formation in coupled laser networks," Phys. Rev. Lett. 106, 223901 (2011).

${ }^{17}$ J. Tiana-Alsina, K. Hicke, X. Porte, M. C. Soriano, M. C. Torrent, J. García-Ojalvo, and I. Fischer, "Zero-lag synchronization and bubbling in delay-coupled lasers,” Phys. Rev. E 85, 026209 (2012).

${ }^{18}$ G. D. Vanwiggeren and R. Roy, "Communication with chaotic lasers," Science 279, 1198-1200 (1998).

${ }^{19}$ A. Argyris, D. Syvridis, L. Larger, V. Annovazzi-Lodi, P. Colet, I. Fischer, J. García-Ojalvo, C. R. Mirasso, L. Pesquera, and K. A. Shore, "Chaos-based communications at high bit rates using commercial fiberoptic links," Nature 438, 343 (2005).

${ }^{20}$ L. Larger and J. P. Goedgebuer, "Cryptography using optical chaos," C. R. Phys. 5, 609 (2004).

${ }^{21}$ A. Uchida, K. Amano, M. Inoue, K. Hirano, S. Naito, H. Someya, I. Oowada, T. Kurashige, M. Shiki, S. Yoshimori, K. Yoshimura, and P. Davis, "Fast physical random bit generation with chaotic semiconductor lasers," Nature Photon. 2, 728-732 (2008).

${ }^{22} \mathrm{C}$.-W. Shih, "Influence of boundary conditions on pattern formation and spatial chaos in lattice systems," SIAM J. Appl. Math. 61, 335-368 (2000).

${ }^{23}$ G. Giacomelli and A. Politi, "Relationship between delayed and spatially extended dynamical systems," Phys. Rev. Lett. 76, 2686 (1996).

${ }^{24}$ O. D'Huys, I. Fischer, J. Danckaert, and R. Vicente, "Spectral and correlation properties of rings of delay-coupled elements: Comparing linear and nonlinear systems,” Phys. Rev. E 85, 056209 (2012).

${ }^{25}$ R. Vicente, J. Daudén, P. Colet, and R. Toral, "Analysis and characterization of the hyperchaos generated by a semiconductor laser subject to a delayed feedback loop,” IEEE J. Quantum Electron. 41, 541 (2005).

${ }^{26} \mathrm{~K}$. Kanno and A. Uchida, "Consistency and complexity in coupled semiconductor lasers with time-delayed optical feedback," Phys. Rev. E 86, 066202 (2012).

${ }^{27}$ S. Heiligenthal, T. Dahms, S. Yanchuk, T. Jüngling, V. Flunkert, I. Kanter, E. Schöll, and W. Kinzel, "Strong and weak chaos in nonlinear networks with time-delayed couplings," Phys. Rev. Lett. 107, 234102 (2011).

${ }^{28}$ S. Heiligenthal, T. Jüngling, O. D’Huys, D. A. Arroyo-Almanza, M. C. Soriano, I. Fischer, I. Kanter, and W. Kinzel, "Strong and weak chaos in networks of semiconductor lasers with time-delayed couplings," Phys. Rev. E 88, 012902 (2013). 